DOI: 10.12731/2227-930X-2021-11-1-59-76 УДК 624.145.72

\title{
МЕТОДЫ ИЗМЕРЕНИЯ ГЛУБИНЫ ЛЕДОВОЙ АБРАЗИИ ПОВЕРХНОСТИ БЕТОННЫХ ОБРАЗЦОВ
}

\author{
Анохин П.В., Беккер А.Т., Уварова Т.Э., \\ Зверев А.А., Беляева Т.Д.
}

Шельф мирового океана богат запасами углеводородного сырья. Большая часть шельфа России приходится на моря арктического и дальневосточного бассейнов, где при проектировании морских ледостойких платформ, грузовых терминалов и портов, необходимо учитыввать суровые климатические условия районов строительства и уделять особое внимание нагрузкам и воздействиям от дрейфующего ледяного покрова. Результатом взаимодействия дрейфующих ледяных полей с гидротехническими сооружениями является абразивный износ поверхности конструкции (бетона). Проблема ледовой абразии строительных материалов привлекает к себе повышенное внимание ученых многих стран. В рамках исследования данного явления проводится множество экспериментов, где методика измерения глубины ледовой абразии может внести значительную погрешность в результаты эксперимента, что способствует необходимости применения новых методов измерения и совершенствования существующих. Так, в Норвежском университете естественных и технических наук за всё время экспериментальных исследований применялась обработка с помощью ичифрового микрометра Mitutoyo Corp. 543270B, 3D оптическим световом сканером ATOS III SO и лазерным сканнером. В Дальневосточном федеральном университете измерения проводились с помощью микрометрического индикатора часового типа (ИЧ) ГОСТ 577-68 (2002) и ичиррового микрометра. В статье приведено описание методов измерения глубины ледовой абразии и даны характеристики измерительного оборудования, 
предложен метод измерения глубинь ледовой абразии с применением оборудования с числовым программным управлением.

Ключевые слова: ледовая абразия бетона; абразия; методы измерения; истирание

\section{METHODS FOR MEASURING THE DEPTH OF ICE ABRASIA ON THE SURFACE OF CONCRETE SAMPLES}

\section{Anohin P.V., Bekker A.T., Uvarova T.E., Zverev A.A., Belyaeva T.D.}

The shelf of the world ocean is rich in hydrocarbon reserves. Most of the Russian shelf is located in the Arctic and Far Eastern seas, where the harsh climatic conditions of the construction areas must be taken into account when designing offshore ice-resistant platforms, cargo terminals and ports, and designers must pay special attention to the loads and impacts from the drifting ice cover. The result of the interaction of drifting ice fields with hydraulic structures is the abrasive wear of the structure surface (concrete). The problem of ice abrasion of building materials attracts the attention of scientists in many countries. As part of the study of this phenomenon, many experiments are conducted, where the method of measuring the ice abrasion depth may bring a significant error in the results of the experiment, which contributes to the need to apply new measurement methods and improve existing ones. For example, at the Norwegian University of Natural and Technical Sciences, Mitutoyo Corp. digital micrometer, 3D optical light scanner ATOS III SO and laser scanner processing were used throughout the experimental studies. At the Far Eastern Federal University, measurements were carried out using a micrometer watch type indicator (WTI) GOST 577-68 (2002) and a digital micrometer. The article describes the methods of measuring the ice abrasion depth and gives the characteristics of measuring equipment, a method for measuring the of ice abrasion depth with numerical control equipment is proposed.

Keywords: concrete ice abrasion; abrasion; measuring methods; wear 


\section{Введение}

В последние годы проблема ледовых истирающих воздействий активно изучается, это обусловлено необходимостью освоения арктического шельфа как с целью добычи углеводородного сырья, так и с целью строительства новых портов и грузовых терминалов для отгрузки и транспортировки этого сырья потребителю. Проектирование, строительство и эксплуатация гидротехнических сооружений в арктических условиях связано с конструированием бетонов повышенной износостойкости. Для этого тщательно изучают процессы ледовой абразии этих бетонов на специально разработанных для этой цели установках [1], [3], [5, с. 183].

В нормативной литературе отсутствуют требования к износостойкости материала, подверженному процессу истирания, что обусловлено сложностью процессов разрушения льда при взаимодействии с сооружением и многообразием характеристик льда.

Целью работы является предложить метод определения глубины ледовой абразии бетона, который должен быть экономичным, простым в применении, малозатратным по времени и обладал достаточной степенью точности измерения.

Для достижения поставленной цели был выполнен литературный обзор методик измерения глубины ледовый абразии. На основании сравнительного анализа существующих методик был разработан новый метод, удовлетворяющий вышеописанным критериям.

\section{Обзор существующих методов определения глубины ледовой абразии бетона}

В статье сравниваются методы измерения глубины ледовой абразии двух научных школ Дальневосточного федерального университета, Россия и Норвежского университета естественных и технических наук, Норвегия, которые принимают активное участие в изучении этого явления и представляют подробные отчеты по использованным методикам и результатам экспериментов.

В России основные экспериментальные исследования сосредоточены в лаборатории МНОЦ «Арктика» Дальневосточного фе- 
дерального университета. В начале исследований для измерения глубины ледовой абразии использовалась установка [2], [6, с. 73], состоящая из координатного стола, прикрепленного к массивной базе, штатива и индикатора часового типа (рисунок 1).

Координатный стол состоит из верхнего и нижнего стола, которые могут перемещаться во взаимно-перпендикулярных направлениях и снабжены линейками для измерений перемещений. Верхний стол с болтами для фиксации образца прикреплен к нижнему столу, а нижний стол - к базе, на которой в определенном положении закреплен штатив с индикатором, и это положение остается постоянным для определения индикатором условного нуля в точке отсчета. Индикатор часового типа изготовлен по ГОСТ 577-68, имеет цену деления 0,01 мм, ход его измерительной иглы составляет $1 \mathrm{~cm}$, погрешность всего прибора - 0,005 мм, а погрешность измерений - 0,05 мм.

С помощью линеек устанавливается точка отсчета условного нуля, затем на верхнем столе устанавливается образец и фиксируется по трем точкам. Измерительную иглу опускают на поверхность измерения и устанавливают шкалу индикатора на ноль.

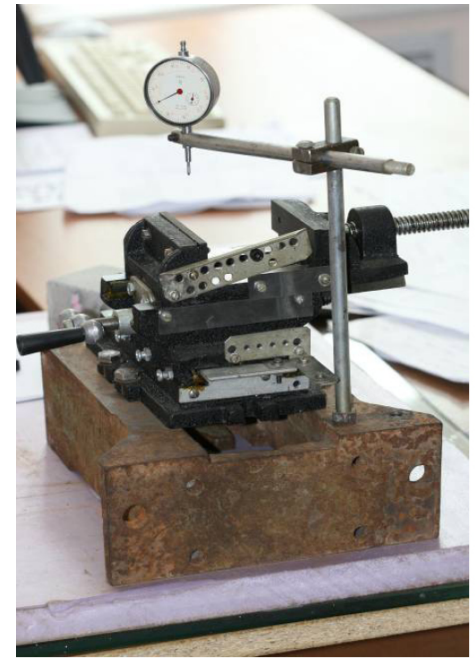

Рис. 1. Измерительная установка с индикатором часового типа

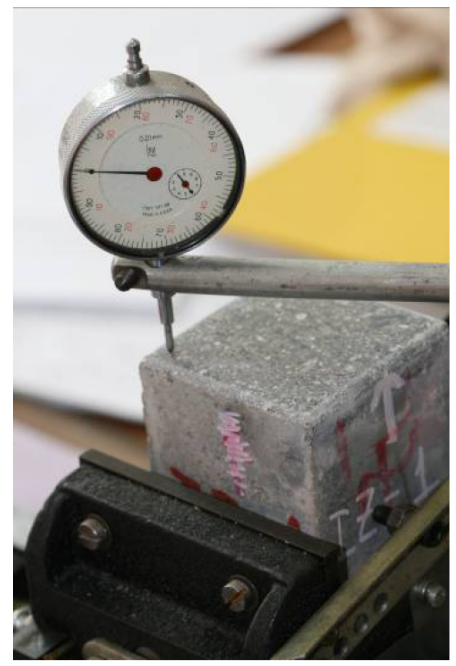

Рис. 2. Фото измерения образца индикатором часового типа 
Когда начальное положение установлено, начинается измерение: иглу индикатора приподнимают и перемещают бетонный образец по осям X и Y с помощью вращения ручек. Измерение проводят дважды - до и после истирания, используя схему с положением 168 точек и направлением движения иглы индикатора (рисунки 3 и 4).

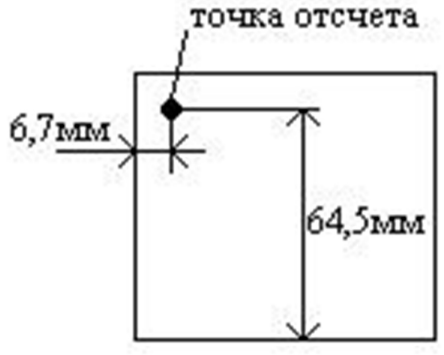

Рис. 3. Точка отсчета иглы индикатора

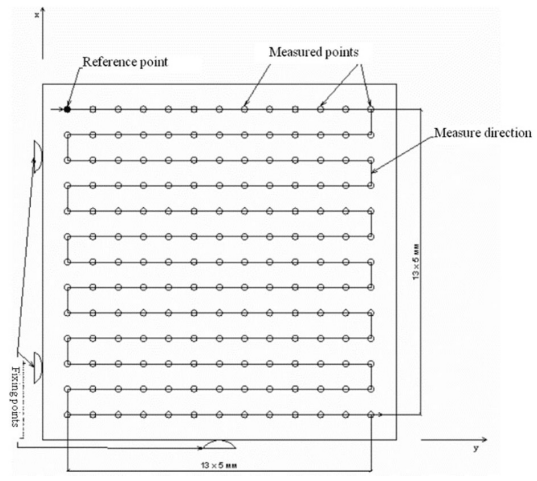

Рис. 4. Положение измеряемых точек и направление хода измерений

После измерения поверхности в программе SURFER строилось изображение истертой поверхности бетонного образца по трем координатами точек поверхности (рисунок 5) [2], [4].

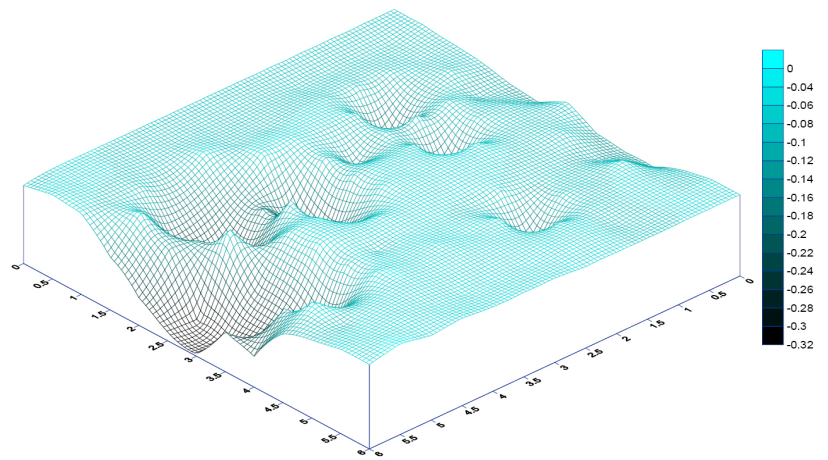

Рис. 5. Пример изображения истертой поверхности, построенного в SURFER [2] 
В Норвегии в начале 2010-х годов для измерения ледовой абразии использовался метод с использованием цифрового микрометра Mitutoyo Corp. 543-270B с разрешением 0,001 мм и указанной точностью 0,003 мм [9], [10], [7, с. 31]. На координатной сетке, разработанной для данного метода, располагалось 110 точек, как указано на рисунке 6.

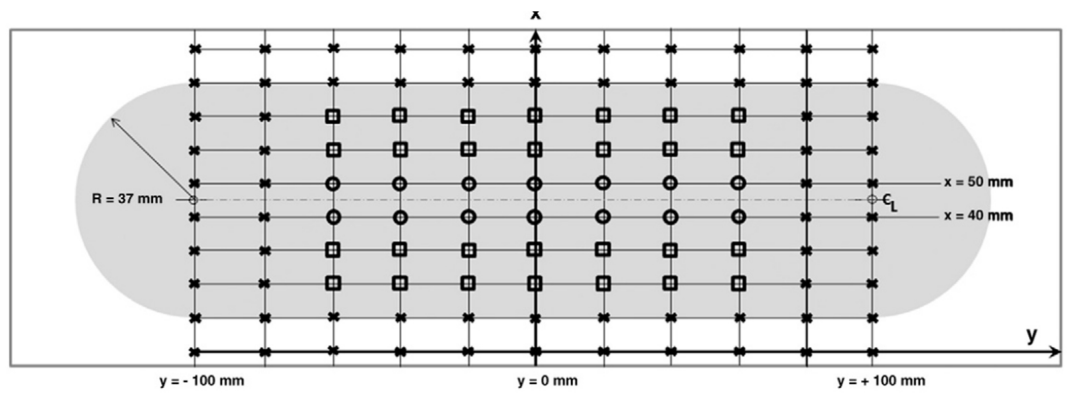

Рис. 6. Распределение точек для измерения глубины ледовой абразии [9], [10]

Глубина истирания измерялась в общей сложности трижды для каждого образца. Точки измерения по линиям $\mathrm{x}=0$ мм и $\mathrm{x}=90$ мм располагались вне зоны воздействия льда и служили исходными точками для измерений абразивного износа. Чтобы избежать воздействия со стороны участков бетонного образца с низкой подверженностью абразии (их наличие обусловлено спецификой экспериментального оборудования, которое описывается в [9]), в качестве основы для оценки скорости истирания были выбраны две подгруппы точек измерения, а именно измерения, помеченные символами $\square$ и ०.

С продолжением экспериментальных исследований требовалось совершенствовать оборудование для получения более полной информации об истертой поверхности бетона, для чего был использован 3D оптический световой сканер ATOS III SO (рисунок 7), который позволял получать цифровые изображения бетонных образцов [7, с. 29], [15], [8]. Он оснащен двумя фотокамерами и проектором, расстояние между фотокамерами было необходимо 
для создания трёхмерного изображения. Шаг между точками измерения для этого сканнера составлял 0,05 мм, а для последующей интерполяционной обработки применялось программное обеспечение ATOS и GOM Inspect.
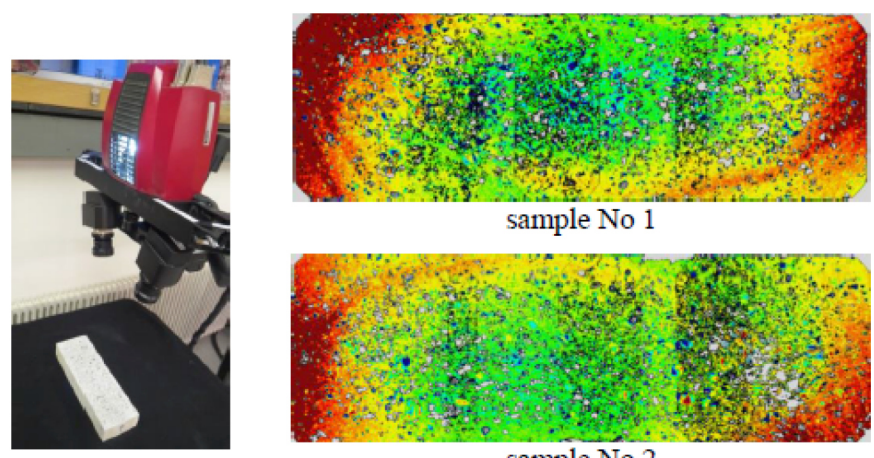

sample No 1
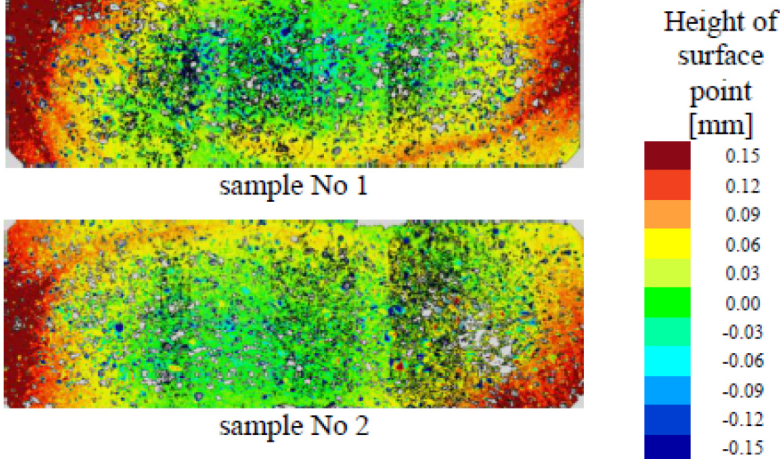

Рис. 7. 3D оптический световой сканер ATOS III SO [15]

Лазерный сканнер с высоким разрешением стал следующим шагом в изучении ледовой абразии (рисунок 8). Лазерный сканер представляет собой комбинацию лазерного датчика, системы линейного перемещения с компьютерным управлением и системы сбора данных, обработки сигналов, обработки данных и графического представления[11], [12], [13], [14]. Лазер движется по образцу в виде «змейки» с разрешением - 0,01 мм, расстояние между точками измерения составляет 50 мкм в направлении оси Y, а расстояние между полосами измерения в направлении оси $\mathrm{X}-1$ мм. Скорость перемещения лазерного сенсора составляла 10 мм/с. Поверхность измерения бетонного образца представляет собой матрицу с общим количеством точек 570 000. Выходные данные составляют значения высоты точек поверхности (в направлении оси Z) вдоль всего пути сканирования образца. В течение всего процесса сканирования движение лазера выходит за пределы сканируемой поверхности для устранения влияния ускорения, торможения или поворота лазерного датчика. 


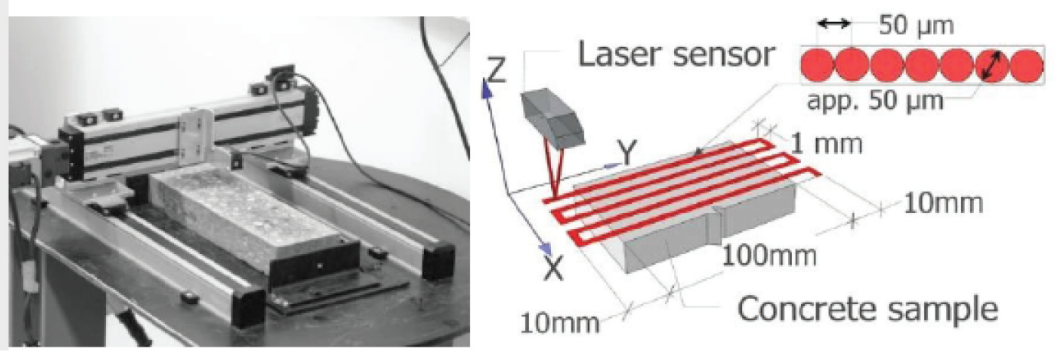

Рис. 8. Лазерный сканнер: фото и упрощенная схема процесса измерения [12]

\section{Сравнительный анализ}

Перечисленные выше методы (таблица 1) имеют свои преимущества и недостатки.

Например, обработка данных с помощью индикатора часового типа оказалась очень сложной, требовала большого количества времени на измерение, а присутствие человеческого фактора значительно увеличивало погрешность измерений. В Норвежском университете естественных и технических наук механическая обработка данных с помощью цифрового микрометра Mitutoyo Corp. 543-270B применялась параллельно с 3D оптическим сканером ATOS III SO для улучшения качества результатов. Однако механический метод обработки уменьшал точность полученных измерений, требовал большого количества времени. После сравнения цифрового датчика и оптического сканера и хорошей согласованности полученных результатов, было решено в дальнейшем отказаться от механического метода.

Однако на изображениях бетонной поверхности, полученных оптическим сканером, были выявлены «черные точки» - не захваченные устройством зоны. Для устранения этих пропущенных зон нужно было проводить повторную съемку бетонной поверхности (1-2 раза) и последующую интерполяционную обработку в программе GOM Inspect. Эти дополнительные меры увеличивали время проведения измерений глубины ледовой 
абразии, поэтому в дальнейшем измерения выполнялись на лазерном сканере.

Он обладал большими преимуществами по сравнению с предыдущим оборудованием, специально для него было разработано программное обеспечение для контроля работы сканера и обработки данных. Такой метод может оказаться очень дорогим для исследователей.

Таблицьа 1.

Сравнительная таблица для методов измерения ледовой абразии

\begin{tabular}{|c|c|c|c|c|}
\hline Устройство & $\begin{array}{c}\text { Индикатор } \\
\text { часового } \\
\text { типа ГОСТ } \\
577-68\end{array}$ & $\begin{array}{c}\text { Mitutoуо Corp. } \\
543-270 \mathrm{~B}\end{array}$ & $\begin{array}{c}\text { 3D оптиче- } \\
\text { ский световой } \\
\text { сканер ATOS } \\
\text { III SO }\end{array}$ & $\begin{array}{c}\text { Лазерный } \\
\text { сканер }\end{array}$ \\
\hline Метод & $\begin{array}{c}\text { Механиче- } \\
\text { ский }\end{array}$ & $\begin{array}{c}\text { Механиче- } \\
\text { ский }\end{array}$ & $\begin{array}{c}\text { Автоматиче- } \\
\text { ский }\end{array}$ & $\begin{array}{c}\text { Автоматиче- } \\
\text { ский }\end{array}$ \\
\hline $\begin{array}{c}\text { Дополнитель- } \\
\text { ное программ- } \\
\text { ное обеспече- } \\
\text { ние }\end{array}$ & $\begin{array}{c}\text { Не требуется } \\
\text { (ручная об- } \\
\text { работка) }\end{array}$ & $\begin{array}{c}\text { Не требуется } \\
\text { ручная обра- } \\
\text { ботка) }\end{array}$ & $\begin{array}{c}\text { Требуется } \\
\text { (ATOS, GOM } \\
\text { Inspect) }\end{array}$ & $\begin{array}{c}\text { Требуется } \\
\text { (специально } \\
\text { разработан- } \\
\text { ное НТНУ) }\end{array}$ \\
\hline $\begin{array}{c}\text { Количество } \\
\text { точек поверх- } \\
\text { ности }\end{array}$ & 168 & 110 & $\begin{array}{c}\text { Около } \\
300000\end{array}$ & 570 000 \\
\hline $\begin{array}{c}\text { Точность при- } \\
\text { бора }\end{array}$ & 0,005 мм & 0,003 мм & 0,05 мм & 0,01 мм \\
\hline
\end{tabular}

В рамках экспериментальных исследований в Дальневосточном федеральном университете была разработана методика измерения глубины ледовой абразии с применением оборудования с числовым программным управлением (ЧПУ).

Оборудование состоит из измерительной машины, датчика измерения и устройства фиксации образца. Измерительная машина (рисунок 9) представляет собой координатный станок с числовым программным управлением и автоматически перемещает датчик измерения. Датчик измерения (рисунок 10) - цифровой микрометр с погрешностью 0,003 мм, погрешность измерительной машины - 0,05 мм. 


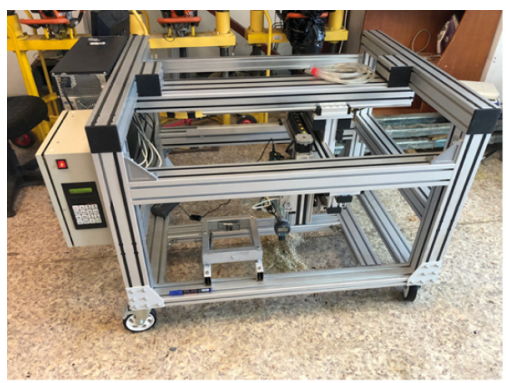

Рис. 9. Общий вид измерительного оборудования

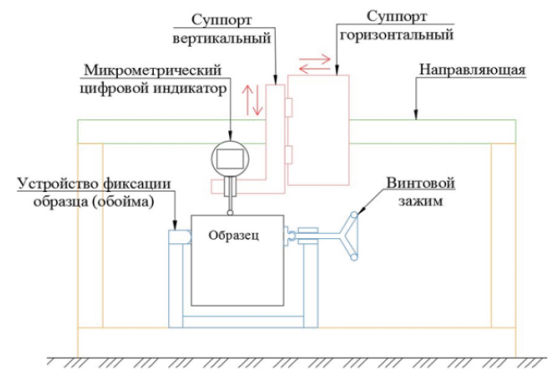

Рис. 10. Схема измерительного оборудования

Бетонный образец фиксируется на установке с помощью устройства фиксации, чтобы фиксировать образец и уменьшить погрешность в горизонтальном направлении. Область измерения ограничивается размерами 131 мм 141 мм и содержит 2160 точек. Исходное положение, откуда начинает двигаться цифровой датчик, помечается пометкой на поверхности - маркером, при этом на образце может быть от 1 до 6 маркеров одновременно (рисунок 11). Результирующая погрешность каждого измерения составляет 0,003 мм.

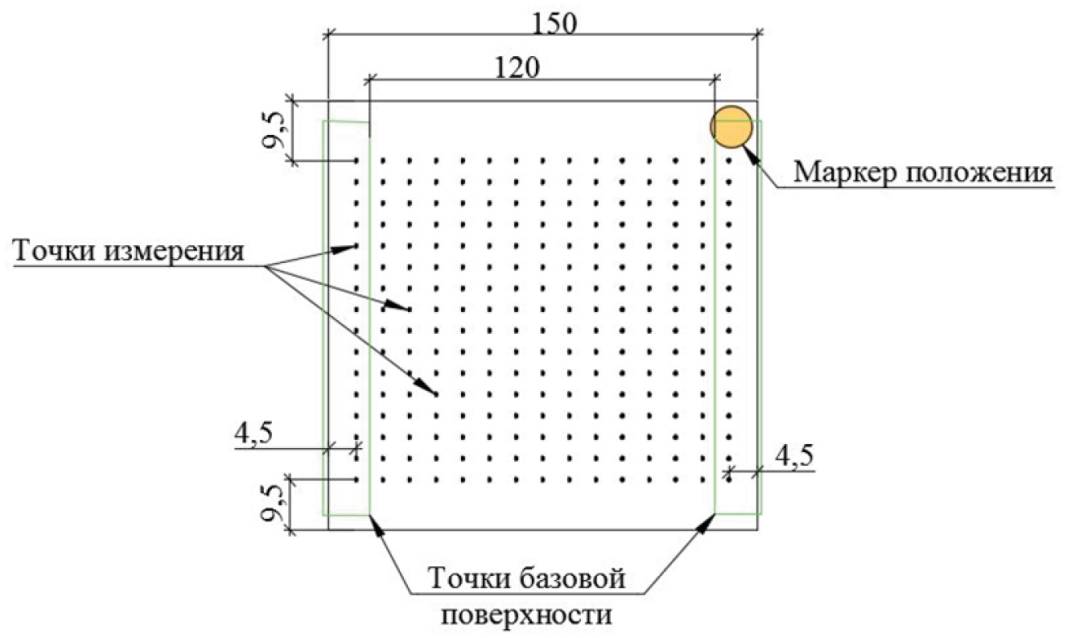

Рис. 11. Область измерения поверхности 
Для обработки данных, полученных в результате измерений, используется программное обеспечение Microsoft Office, MathCAD и MATLAB. B MathCAD решается проблема смещения бетонного образца в вертикальном направлении при установке его на измерительное оборудование. Для этого используется привязка плоскости измерения к базовой плоскости, которая создается через точки поверхности, свободных от погрешности, и уравнения плоскости.

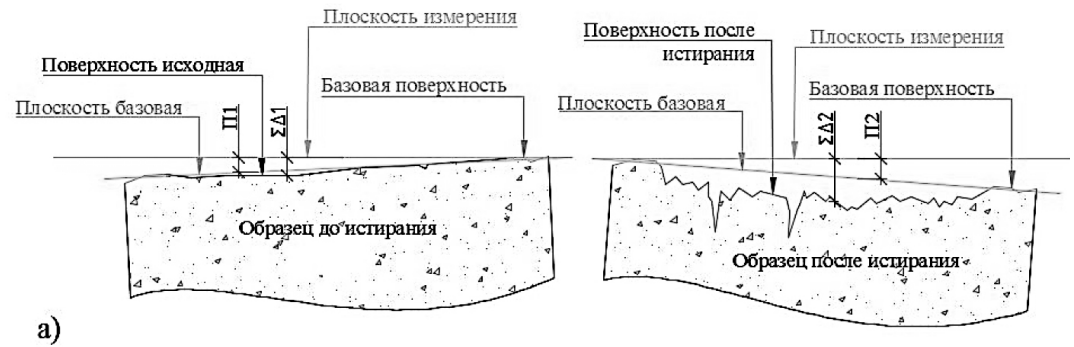

a)

б)

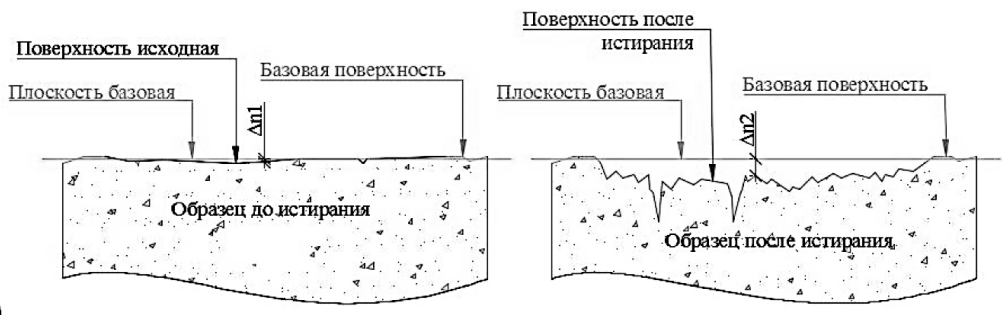

Рис. 12. Измеряемая поверхность образца а) до привязки к базовой поверхности б) после привязки к базовой поверхности

Разница между плоскостью измерения и базовой плоскостью ошибка, которую определяет погрешность процесса измерения. На рисуноке 12 показаны отклонения положения образца «П1» и «П2», «П1»- до начала истирания, «П2»- после. Обычно ошибка измерения состоит из вертикального смещения образца и его наклона, то есть, из постоянной и переменной составляющей. Корректировка измерений каждой точки на величины «П1» и «П2» позволяет получить данные, свободные от погрешности: 


$$
\left\{\begin{array}{l}
\Delta n 1_{i}=\Sigma \Delta 1_{i}-\Pi 1_{i} \\
\Delta n 2_{i}=\Sigma \Delta 2_{i}-\Pi 2_{i}
\end{array}\right.
$$

где $\Delta n 1$ и $\Delta n 2$ - значения отклонения поверхности до и после истирания образца, $\sum n 1$ и $\sum n 2-$ результаты измерения глубины ледовой абразии до и после эксперимента.

Уравнение, приведенное к общей базе измерения, примет вид:

$$
\Delta_{\mathrm{cp}}=\frac{1}{N} \times \sum_{i=0}^{N}\left(\Delta n 1_{i}-\Delta n 2_{i}\right) .
$$

где $N$ - количество точек на поверхности.

Обработка и подготовка данных для дальнейшего использования проводится в MathCAD с последующим сохранением массива значений результатов измерений в формате Excel. Первоначальная оценка результатов истирания проводится по изображениям, построенным в электронных таблицах, и представляет собой поле точек, где цветом обозначается глубина истёртой поверхности (рисунок 13).

MATLAB используется для написания кода, который подробно обрабатывает файлы с измерениями поверхности и выдает общую таблицу с результатами по глубине ледовой абразии и исходными данными для каждого образца соответственно. Для удобства в этой программе прописывается дополнительный код для выведения изображения истертой поверхности бетонного образца (рисунок 14).

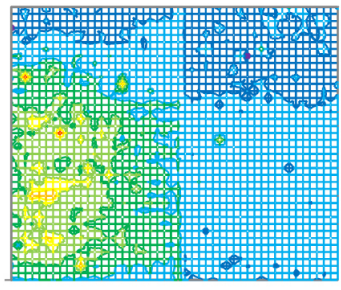

Рис. 13. Изображение истертой поверхности в Microsoft Excel

\author{
Величина \\ износа, \\ $\mathrm{MM}$ \\ $\square 1-1,2$ \\ $\square 0,8-1$ \\ $\square, 6-0,8$ \\ $\square 0,4-0,6$ \\ $\square 0,2-0,4$ \\ $\square 0-0,2$ \\ $\square-0,2-0$ \\ $\square-0,4--0,2$
}

\section{Выводы}

Несмотря на дополнительное использование специально разработанных программ для обработки экспериментальных данных, метод с использованием устройства с ЧПУ является экономически 
оптимальным решением. Во-первых, перечисленные программы широко используются в различных организациях. Во-вторых, коды в MATLAB и в MathCAD пишутся однократно, далее обработка от получения файлов с измерительного оборудования до конечной результирующей таблицы занимает около 5 минут. Всё это делает предложенную методику достаточно простой, легко воспроизводимой, понятной. Дополнительным преимуществом является невысокая стоимость оборудования и минимизация времени проведения измерений.

Методика представляет собой законченных комплекс программного обеспечения и оборудования для измерения глубины ледовой абразии поверхности образцов и была апробирована в процессе проведения экспериментальных исследований по истиранию бетона льдом [6].

\section{Список литературь}

1. Bekker A.T., Uvarova T.E., Pomnikov E.E. Calculation of ice abrasion for the lighthouses installed in the Gulf of Bothnia // Proceedings of the 21-th International Offshore and Polar Engineering Conference. Montréal, Canada, 2011, pp. 273-281.

2. Bekker A.T., Uvarova T.E., Pomnikov E.E., Farafonov A.E., Prytkov I.G., Tyutrin R.S. Experimental Study of Concrete Resistance to Ice Abrasion // Proceedings of the 21-th International Offshore and Polar Engineering Conference Maui, Hawaii, 2011, pp. 1044-1047

3. Jacobsen S., Kim L.V., Pomnikov E.E. Concrete Destructure due to Ice-Indentation Pore Pressure // Proceedings of the 21-th International Offshore and Polar Engineering Conference. Rhodes, Greece, June 1722, 2012, pp. 1258-1263.

4. Jacobsen S., Bekker A.T., Uvarova T.E., Pomnikov E.E., Kim L.V., Fosså K.T., Concrete Ice Abrasion: Deterioration Mechanisms, Testing and Modeling // Proceedings ICDC 2012 International Congress on Durability of Concrete, Trondheim Norway, June 18-21.

5. Уварова Т.Э. Истирающее воздействие дрейфующего ледяного покрова на морские гидротехнические сооружения: диссертация на 
соискание ученой степени доктора технических наук. Владивосток, 2015. $271 \mathrm{c}$.

6. Фисенко В. П., Уварова Т. Э., Сравнительный анализ методик испытаний строительных материалов на ледовую абразию // Актуальные исследования. 2020. №11(14). С. 68-74.

7. Bøhn O. D. Ice abrasion of concrete, background theory and testing at the NTNU laboratory: master thesis. Trondheim, 2012. 67 p.

8. Jacobsen S., Scherer G. W., Schulson E. M., Concrete-ice abrasion mechanics // Cement and Concrete Research, 2015, vol. 73, pp. 79-95.

9. Møen E., Høiseth K. V., Leira B., Høyland K. V., Experimental study of concrete abrasion due to ice friction. Part I: Set-up, ice abrasion vs. material properties and exposure conditions // Cold Regions Science and Technology, 2015, vol. 110, pp. 183-201.

10. Møen E., Høiseth K. V., Leira B., Høyland K. V., Experimental study of concrete abrasion due to ice friction. Part II: Statistical representation of abrasion rates and simple, linear models for estimation // Cold Regions Science and Technology, 2015, vol. 110, pp. 202-214.

11. Shamsutdinova G., Hendriks M.A.N., Jacobsen S., Concrete ice abrasion laboratory experiments // Proceedings of 24-th International Conference on Port and Ocean Engineering under Arctic Conditions, Busan, Korea, 2017, June 11-16.

12. Shamsutdinova G., Hendriks M.A.N., Jacobsen S., Concrete-ice abrasion test with sliding ice and ice spallation // Nordic Concrete Research. 2017, vol. 57, pp. 39-57.

13. Shamsutdinova G., Hendriks M.A.N., Jacobsen S., Concrete-ice abrasion: wear, coefficient of friction and ice consumption // Wear, 2018. no. 416-417, pp. 27-35.

14. Shamsutdinova G., Hendriks M.A.N., Jacobsen S., Topography studies of concrete abraded with ice // Wear, 2019. no. 430-431, pp. 1-11.

15. Shamsutdinova G., Rike P. B., Hendriks M.A.N., Jacobsen S., Concrete ice abrasion rig and wear measurements // Proceedings of 23-th International Conference on Port and Ocean Engineering under Arctic Conditions, Trondheim, Norway, 2015, June 14-18. 


\section{References}

1. Bekker A.T., Uvarova T.E., Pomnikov E.E. Calculation of ice abrasion for the lighthouses installed in the Gulf of Bothnia. Proceedings of the 21-th International Offshore and Polar Engineering Conference. Montréal, Canada, 2011, pp. 273-281.

2. Bekker A.T., Uvarova T.E., Pomnikov E.E., Farafonov A.E., Prytkov I.G., Tyutrin R.S. Experimental Study of Concrete Resistance to Ice Abrasion. Proceedings of the 21-th International Offshore and Polar Engineering Conference Maui, Hawaii, 2011, pp. 1044-1047

3. Jacobsen S., Kim L.V., Pomnikov E.E. Concrete Destructure due to Ice-Indentation Pore Pressure. Proceedings of the 21-th International Offshore and Polar Engineering Conference. Rhodes, Greece, June 17-22, 2012, pp. 1258-1263.

4. Jacobsen S., Bekker A.T., Uvarova T.E., Pomnikov E.E., Kim L.V., Fosså K.T., Concrete Ice Abrasion: Deterioration Mechanisms, Testing and Modeling. Proceedings ICDC 2012 International Congress on Durability of Concrete, Trondheim Norway, June 18-21.

5. Uvarova T. E. Istirayushchee vozdeystvie dreyfuyushchego ledyanogo pokrova na morskie gidrotekhnicheskie sooruzheniya [Abrasive drift effects ice cover on marine hydraulic structures]. Vladivostok, 2015, $271 \mathrm{p}$.

6. Fisenko V. P., Uvarova T. E., Sravnitel'nyy analiz metodik ispyta-niy stroitel'nykh materialov na ledovuyu abraziyu [Comparative analysis of building materials testing methods for ice abrasion]. Aktual'nye issledovaniya, 2020, no. 11(14), pp. 68-74.

7. Bøhn O. D. Ice abrasion of concrete, background theory and testing at the NTNU laboratory: master thesis. Trondheim, 2012. $67 \mathrm{p}$.

8. Jacobsen S., Scherer G. W., Schulson E. M., Concrete-ice abrasion mechanics. Cement and Concrete Research, 2015, vol. 73, pp. 79-95.

9. Møen E., Høiseth K. V., Leira B., Høyland K. V., Experimental study of concrete abrasion due to ice friction. Part I: Set-up, ice abrasion vs. material properties and exposure conditions. Cold Regions Science and Technology, 2015, vol. 110, pp. 183-201.

10. Møen E., Høiseth K. V., Leira B., Høyland K. V., Experimental study of concrete abrasion due to ice friction. Part II: Statistical representa- 
tion of abrasion rates and simple, linear models for estimation. Cold Regions Science and Technology, 2015, vol. 110, p. 202-214.

11. Shamsutdinova G., Hendriks M.A.N., Jacobsen S., Concrete ice abrasion laboratory experiments. Proceedings of 24-th International Conference on Port and Ocean Engineering under Arctic Conditions, Busan, Korea, 2017, June 11-16.

12. Shamsutdinova G., Hendriks M.A.N., Jacobsen S., Concrete-ice abrasion test with sliding ice and ice spallation. Nordic Concrete Research, 2017, vol. 57, pp. 39-57.

13. Shamsutdinova G., Hendriks M.A.N., Jacobsen S., Concrete-ice abrasion: wear, coefficient of friction and ice consumption. Wear, 2018, no. 416-417, pp. 27-35.

14. Shamsutdinova G., Hendriks M.A.N., Jacobsen S., Topography studies of concrete abraded with ice. Wear, 2019, no. 430-431, pp. 1-11.

15. Shamsutdinova G., Rike P. B., Hendriks M.A.N., Jacobsen S., Concrete ice abrasion rig and wear measurements. Proceedings of 23-th International Conference on Port and Ocean Engineering under Arctic Conditions, Trondheim, Norway, 2015, June 14-18.

\section{ДАННЫЕ ОБ АВТОРАХ}

Анохин Павел Викторович, заведующий лабораторией МНОЦ «Арктика» департамента «Морские арктические технологии»

Дальневосточный федеральный университет n. Аякс, 10, о. Русский, г. Владивосток, Приморский край, 690922, Российская Федерацияя.

anokhin.pv@dvfu.ru

Беккер Александр Тевьевич, профессор департамента «Морские арктические технологии», доктор технических наук Дальневосточный федеральный университет n. Аякс, 10, о. Русский, г. Владивосток, Приморский край, 690922, Российская Федерация.

bekker.at@dvfu.ru 
Уварова Татьяна Эриковна, профессор департамента «Морские арктические технологии», доктор технических наук Дальневосточный федеральный университет n. Аякс, 10, о. Русский, г. Владивосток, Приморский край, 690922, Российская Федераџия.

uvarova.tye@dvfu.ru

Зверев Антон Андреевич, ассистент департамента «Морские арктические технологии»

Дальневосточный федеральный университет n. Аякс, 10, о. Русский, г. Владивосток, Приморский край, 690922, Российская Федеращия.

zverev.aa@dvfu.ru

Беляева Тамара Дмитриевна, инженер-исследователь МНОЦ «Арктика», магистрант «Offshore and Coastal Engineering» департамента «Морские арктические технологии»

Дальневосточный федеральный университет

n. Аякс, 10, о. Русский, г. Владивосток, Приморский край, 690922, Российская Федерация.

beliaeva.tdm@dvfu.ru

\section{DATA ABOUT THE AUTHORS}

Pavel V. Anohin, Head of the Laboratory ISEC «Arctic» of Department «Marine Arctic Technologies»

Far Eastern Federal University

10, Ajaks St., Russky Island, Vladivostok, Primorski krai, 690922, Russian Federation.

anokhin.pv@dvfu.ru

Aleksandr T. Bekker, Professor of Department «Marine Arctic Technologies», Doctor of Technical Science

Far Eastern Federal University

10, Ajaks St., Russky Island, Vladivostok, Primorski krai, 
690922, Russian Federation.

bekker.at@dvfu.ru

Tatiana E. Uvarova, Professor of Department «Marine Arctic Technologies», Doctor of Technical Science

Far Eastern Federal University

10, Ajaks St., Russky Island, Vladivostok, Primorski krai, 690922, Russian Federation.

uvarova.tye@dvfu.ru

Anton A. Zverev, Assistant of Department «Marine Arctic Technologies» Far Eastern Federal University

10, Ajaks St., Russky Island, Vladivostok, Primorski krai, 690922, Russian Federation.

zverev.aa@dvfu.ru

Tamara D. Belyaeva, Research Engineer of ISEC «Arctic», Master Student of «Offshore and Coastal Engineering» of Department «Marine Arctic Technologies»

Far Eastern Federal University

10, Ajaks St., Russky Island, Vladivostok, Primorski krai, 690922, Russian Federation.

beliaeva.tdm@dvfu.ru 